\title{
Complex pp waves and the nonlinear graviton construction
}

\author{
W. D. Curtis \\ Department of Mathematics, Kansas State University, Manhattan, Kansas 66506 \\ D. E. Lerner \\ Department of Mathematics, University of Kansas, Lawrence, Kansas 66045 \\ F. R. Miller
}

Department of Mathematics, Kansas State University, Manhattan, Kansas 66506

(Received 12 April 1978)

We show how to construct all of the complex $p p$ waves using the nonlinear graviton construction of Penrose.

\section{INTRODUCTION}

Several years ago, Penrose ${ }^{1}$ showed that one could construct generic, half-flat solutions of the complex Einstein equations through the use of deformation theory. His idea is to deform the complex structure of a neighborhood of a projective line in $P_{3}(C)$. The original undeformed neighborhood contains a four-complex-parameter family of lines which are identified with the points of an open subset of complex Minkowski space. For small deformations, Kodaira's theorems $s^{2,3}$ guarantee the continued existence of a four-parameter family of "lines" ( $i, e_{.}$, compact holomorphic curves) in the deformed space, these are identified as the points of a new complex manifold $G$. A holomorphic metric is then introduced on $G$ in a natural way and the resulting complex spacetime is shown to be half-flat, that is, its Ricci tensor vanishes and its conformal curvature tensor is anti-self-dual.

While it is a relatively straightforward matter to construct deformations, the task of finding the fourparameter family of lines in the deformed space is usually very difficult. Because of this, only a few isolated solutions have actually been explicitly constructed, only one of which, to our knowledge, has appeared in print. ${ }^{4}$ The purpose of this paper is to show that the simplest half-flat spacetimes, known as complex pp waves or Plebański plane waves, ${ }^{5}$ can all be obtained explicitly using the Penrose construction

\section{THE NONLINEAR GRAVITON CONSTRUCTION}

In this section we summarize the Penrose construction. For more details, in particular for the proof that $G$ is half-flat, we refer the reader to Penrose's original article. ${ }^{1}$

Denote a point of $C^{4}-(0)$ (a twistor) by $Z^{\alpha}=\left(\omega^{A}, \pi_{A^{\prime}}\right)$, and let $\left[\omega^{A}, \pi_{A^{*}}\right]$ denote the corresponding point in $P_{3}(C)$. If $x^{A A^{\prime}}$ is any point in complex Minkowski space, $\mathrm{CM}$, we may associate with it the projective line $L(x)$ : $=\left\{\left[i x^{A A^{\prime}} \pi_{A^{\prime}}, \pi_{A^{\prime}}\right] \mid\left[\pi_{A^{\prime}}\right] \subseteq P_{1}(C)\right\}$. If $W$ is a connected open neighborhood of $x$ in CM, the set $P T(W):=\{L(y) \mid y \in W\}$ is a connected open neighborhood of $L(x)$ in $P_{3}(C)$. In this paper we shall consider only the case $W=\mathrm{CM}$ and we set $P T:=P T(\mathrm{CM})$. Notice that $P T$ is just $P_{3}(C)$ with one projective line removed (namely, all points of the form $\left.\left[\omega^{A}, 0\right]\right) 。 T$ is the corresponding set of points in $C^{4}-(0)$. Then we have:

(a) $P T$ is a holomorphic fiber space over $P_{1}(C)$ with projection $\left[\omega^{A}, \pi_{A^{\prime}}\right] \rightarrow\left[\pi_{A^{\prime}}\right]$. Similarly we have a fiber space $T \rightarrow C^{2}-(0)$ given by $\left(\omega^{A}, \pi_{A^{\prime}}\right) \rightarrow \pi_{A^{\prime}}$ and the following diagram commutes.

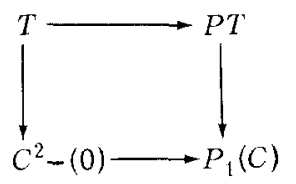

(b) The points of CM are in $1-1$ correspondence with the global holomorphic cross sections of $P T \rightarrow P_{1}(C)$ : Given $x^{A A^{\prime}}$, define a section by $\left[\pi_{A^{\prime}}\right] \rightarrow\left[i x^{A A^{\prime}} \pi_{A^{\prime}} \pi_{A^{\prime}}\right]$. Alternately points of $\mathrm{CM}$ may be put in correspondence with global holomorphic cross sections of $T \rightarrow C^{2}-(0)$ which are homogeneous of degree 1 in $\pi_{A^{\prime}}$.

(c) The conformal structure of $\mathrm{CM}$ is obtained by observing that points $x$ and $y$ in CM are null-separated iff $L(x)$ and $L(y)$ intersect. If $y=x+\Delta x$ and if $\omega^{A}\left(\Delta x, \pi_{A^{\prime}}\right)$ is the section of $T \rightarrow C^{2}-(0)$ corresponding to $\Delta x$, then $\omega^{A}\left(\Delta x, \stackrel{\circ}{\pi}_{A^{\prime}}\right)=0$ for some $\stackrel{\circ}{\pi}_{A^{\prime}}$. [and hence for $\lambda{\stackrel{\circ}{A^{\prime}}}^{\prime}$ for $\lambda C C-(0)]$. Thus null vectors in CM correspond to global holomorphic sections of $T \rightarrow C^{2}-(0)$ which vanish somewhere. In order to pin down the conformal factor, one makes use of the 2 -form $d \omega_{A} \wedge d \omega^{A}=\mu$. This will be considered in more detail later.

If we let $L$ (resp. $\hat{D}$ ) be the subset of $C^{2}-(0)$ given by $\pi_{0^{\prime}} \neq 0\left(\right.$ resp,$\left.\pi_{1^{\prime}} \neq 0\right)$, then we get a decomposition of $T$ as the union $U \cup \hat{U}$, where $U=\left\{\left(\omega^{A}, \pi_{A^{\prime}}\right) \mid \pi_{A^{\prime}}<D_{\uparrow}\right.$ and $\hat{U}=\left\{\left(\hat{\omega}^{A}, \hat{\pi}_{A}\right) \mid \hat{\pi}_{A},-\hat{0}\right\}$. We may view $T$ as being formed by glueing together $\hat{U}$ and $\hat{U}$ by the trivial equations $\hat{\omega}^{A}=\omega^{A}, \hat{\pi}_{A^{\prime}}=\pi_{A^{\prime}}$. To deform $T$, we consider a oneparameter family of patchings of the form

$$
\hat{\omega}^{A}=\hat{F}^{A}(\omega, \pi, \lambda), \quad \hat{\pi}_{A^{\prime}}=\pi_{A^{\prime}},
$$

satisfying

$$
\begin{aligned}
& j(\alpha \omega, \alpha \pi, \lambda)=\alpha j^{A}(\omega, \pi, \lambda), \alpha: C-(0), \\
& \gamma^{A}(\omega, \pi, 0)=\omega^{A} .
\end{aligned}
$$

Here $\lambda$ ranges over a neighborhood $B$ of $0 \subseteq C$ and the 


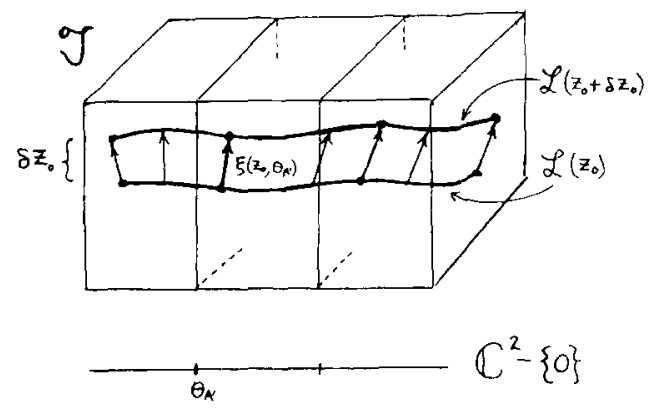

FIG. 1.

functions $\hat{f}^{A}$ are holomorphic in $C^{2} \times(D \cap \hat{D}) \times B$. For each fixed $\lambda \in B$ the patching (2) gives a fiber space $T(\lambda) \rightarrow C^{2}-(0)$ (a deformed twistor space). Since the transition functions are homogeneous of degree 1 , the identification $\left(\omega^{A}, \pi_{A^{\prime}}\right) \sim\left(\alpha \omega^{A}, \alpha \pi_{A^{\prime}}\right), \alpha \in C-(0)$, is consistent over $D \cap \hat{D}$ and gives rise to a deformed projective twistor space $P T(\lambda) \rightarrow P_{1}(C)$. An important aspect of these deformed twistor spaces is the existence of a preferred 2-form on the fibers of $T(\lambda) \rightarrow C^{2}-(0)$; the transformations (2) are required to satisfy $d \hat{\omega}^{0} \wedge d \hat{\omega}^{1}$ $=d \omega^{0} \wedge d \omega^{2}+$ (terms involving $d \pi_{A^{\prime}}$ )。

Kodaira's stability theorem ${ }^{2}$ ensures that for $|\lambda|$ sufficiently small, $P T(\lambda) \rightarrow P_{1}(C)$ still has a global holomorphic section. His completeness theorem ${ }^{3}$ then guar antees the existence of a four-parameter family of global holomorphic sections of $P \bar{T}(\lambda) \rightarrow P_{1}(C)$. So for $\lambda$ fixed and sufficiently small we have an open set $\mathcal{C} \subset C^{4}$ and for each $z \in \mathcal{Y}$ a global holomorphic section, $L_{\lambda}(z)$, of $P T(\lambda)-P_{1}(C)$, distinct $z$ 's giving rise to distinct sections. Each such $L_{\lambda}(z)$ pulls back to a global holomorphic section of $T(\lambda) \rightarrow C^{2}-(0)$ which is homogeneous of degree 1 . This section, $L_{\lambda}(z)$, is represented by a pair $\left\{\omega^{A}(z, \pi, \lambda), \hat{\omega}^{A}(z, \pi, \lambda)\right\}$ satisfying the transition relation (2). Each function $\omega^{A}(z, \pi, \lambda)$, $\hat{\omega}^{A}(z, \pi, \lambda)$ is holomorphic on its domain and homogenneous of degree 1 in $\pi_{A^{\prime}}$.

Henceforth we shall consider a fixed value of $\lambda$ and shall omit $\lambda$ from the notation.

Now let $z_{0} \in \mathcal{G}$ and let $\delta z=\xi^{a}\left(\partial / \partial z^{a}\right)\left(z_{0}\right)$ be tangent to $G$ at $z_{0}$. Define $\xi^{A}\left(z_{0}, \pi\right):=\xi^{a}\left(\partial \omega^{A} / \partial z^{a}\right)\left(z_{0}, \pi\right)$ and $\hat{\xi}^{A}\left(z_{0}, \pi\right):=\xi^{a}\left(\partial \hat{\omega}^{A} / \partial z^{a}\right)\left(z_{0}, \pi\right)$. Then

$$
\hat{\xi}^{A}\left(z_{0}, \pi\right)=\left(\partial \hat{f}^{A} / \partial \omega^{B}\right)\left(\omega\left(z_{0}, \pi\right), \pi\right) \xi^{B}\left(z_{0}, \pi\right) .
$$

Thus the pair $\left\{\xi^{A}, \hat{\xi}^{A}\right\}$ is a section of the normal bundle of $\angle\left(z_{0}\right)$ in $T$. Intuitively the situation is as follows: $\angle\left(z_{0}\right)$ is a section of $T \rightarrow C^{2}-(0)$ which we view as a submanifold of 7 . We write (to first order) $\angle\left(z_{0}+\delta z\right):=\left\{\omega^{A}\left(z_{0}, \pi\right)\right.$ $\left.+\xi^{A}\left(z_{0}, \pi\right) ; \hat{\omega}^{A}\left(z_{0}, \pi\right)+\hat{\xi}^{A}\left(z_{0}, \pi\right)\right\}$.

That is, we have a "nearby" section $\angle\left(z_{0}+\delta z\right)$ and the "difference" between the two is a section of the normal bundle of $\angle\left(z_{0}\right)$ (see Fig. 1). It follows from Kodaira's completeness theorem ${ }^{3}$ that the map $\delta z=\xi^{a}\left(\partial / \partial z^{a}\right)\left(z_{0}\right)$ $\rightarrow \xi\left(z_{0}, \pi\right)$ is an isomorphism from $T_{z_{0}} G$ onto the space of global holomorphic sections of the normal bundle of $L\left(z_{0}\right)$ which are homogeneous of degree 1 in $\pi_{A^{\prime}}$.

The conformal metric on $\mathcal{G}$ is obtained by defining $\xi_{z} \in T_{z_{0}}$ to be null if and only if $\xi(z, \pi)$ has a zero at some $\pi$ (and hence for any nonzero multiple of $\pi$ ). See (c) above.

In order to define the actual metric on $\mathcal{G}$ we use the 2-form, $\mu=\epsilon_{A B} d \omega^{A} \wedge d \omega^{B}=\epsilon_{A B} d \hat{\omega}^{A} \wedge d \hat{\omega}^{B}\left(\bmod d \pi_{A^{\prime}}\right)$, which is well defined on the fibers of $T$. If $\xi \in T, \mathcal{G}$ the relation (4) shows that we may regard $\xi(z, \pi)$ as a vector field along $L(z)$ which is everywhere tangent to the fibers. Suppose $\xi$ and $\eta$ are null vectors at $z$ and that $\xi(z, \pi), \eta(z, \pi)$ vanish at $\alpha_{A^{\prime}}, \beta_{A^{\prime}}$, respectively. Then Penrose defines

$$
g_{z}(\xi, \eta):=\left(\alpha_{B^{\prime}} \beta^{B^{\prime}}\right) \mu(\xi, \eta) /\left(\alpha_{B}, \pi^{B^{\prime}}\right)\left(\beta_{B^{\prime}}, \pi^{B^{\prime}}\right) .
$$

The right side of $(5)$ is symmetric in $(\xi, \eta)$. As a function of $\pi_{A^{\prime}}$, it is homogeneous of degree 0 , and holomorphic on $C^{2}-(0)$. Thus it is constant and so $g_{z}(\xi, \eta)$ is a welldefined complex number.

\section{CONSTRUCTION OF THE COMPLEX pp WAVES}

We choose the patching (2) to have the form

$$
\begin{aligned}
& \hat{\omega}^{0}=\omega^{0}, \\
& \hat{\omega}^{1}=\omega^{1}+\lambda h\left(\omega^{0}, \pi_{A^{\prime}}\right), \\
& \hat{\pi}_{A^{\prime}}=\pi_{A^{\prime}},
\end{aligned}
$$

where $h$ is homogeneous of degree 1 and holomorphic on $C \times(D \cap \hat{D})$. We set $\lambda=1$ in what follows. We shall write down all global holomorphic sections of the bundle $T \rightarrow C^{2}-(0)$ obtained using (6) which are also homogeneous of degree 1 in $\pi_{A^{*}}$. Such a section is given by a pair $\left\{\omega^{A}(\pi), \hat{\omega}^{A}(\pi)\right\}$ satisfying (6) with $\omega^{A}(\pi), \hat{\omega}_{\hat{A}}^{A}(\pi)$ homogeneous of degree 1 and holomorphic in $D, \hat{D}$ respectively. Thus $\omega^{0}(\pi)=\hat{\omega}^{0}(\pi)$, so these give a holomorphic function on $C^{2}-(0)$, homogeneous of degree 1 . Therefore, there exist $u, \zeta \in C$, constants, such that

$$
\hat{\omega}^{0}(\pi)=\omega^{0}(\pi)=u \pi_{0},+\zeta \pi_{1},
$$

$\omega^{1}(\pi)$ and $\hat{\omega}^{1}(\pi)$ are related as follows:

$$
\hat{\omega}^{1}(\pi)=\omega^{1}(\pi)+h\left(\omega^{\circ}(\pi), \pi\right), \quad \pi \in D \cap \hat{D} .
$$

For fixed $u$ and $\xi, h\left(u \pi_{0^{\prime}}+\zeta \pi_{1^{\prime}}, \pi_{0^{\prime}}, \pi_{1},\right)$ is a holomorphic function $D \cap \hat{D}$. To construct a section of our bundle, we must express this function as a difference $\hat{\omega}^{1}(\pi)-\omega^{1}(\pi)$ where $\omega^{1}\left(\hat{\omega}^{1}\right)$ is holomorphic in $D(\hat{D})$ and homogeneous of degree 1. Each distinct way of splitting $h$ will give a pair $\left\{\hat{\omega}^{1}, \omega^{1}\right\}$ and hence a section. We claim that for each pair $(u, \zeta)$ there is a two complex parameter family of splittings. Thus we get a four-parameter family of sections of $T \rightarrow C^{2}(0)$ as desired.

To see this, let $\xi \neq 0$. Then setting $\left(\pi_{0}, \pi_{1},\right)=(1 / \xi, 1)$ in $(8)$,

$$
\hat{\omega}^{1}\left(\xi^{-1}, 1\right)=\xi^{-1} \omega^{1}(1, \xi)+\xi^{-1} h(u+\xi \zeta, 1, \xi) .
$$

Let $h(\xi):=h(u+\xi \xi, 1, \xi)$ and expand $h(\xi)$ in a Laurent series, $h=\sum_{n=-\infty}^{\infty} b_{n}(u, \zeta) \xi^{n}$. Put $\omega^{1}(1, \xi)=\sum_{n=0}^{\infty} a_{n} \xi^{n}$ 。 This series is convergent for all $\xi \in C$, and $\xi^{-1} \omega^{1}(1, \xi)$ $+\xi^{-1} h(\xi)$ is to be entire in $\xi^{-1}$. We conclude that in the series expansion of the right side of (8) all positive 
powers of $\xi$ disappear. We conclude $a_{n}=-b_{n}$ for $n \geqslant 2$, while $\widetilde{\zeta}:=a_{0}$ and $v:=a_{1}$ are free parameters. Thus in the first chart the sections are given by

$\omega^{0}\left(u, v, \zeta, \tilde{\zeta}, \pi_{A^{\prime}}\right)=u \pi_{0^{\prime}}+\zeta \pi_{1}$,

$\omega^{1}\left(u, v, \zeta, \tilde{\zeta}, \pi_{A^{\prime}}\right)=\tilde{\zeta} \pi_{\alpha^{*}}+v \pi_{1^{\prime}}-\sum_{n=2}^{\infty} b_{n}(u, \zeta) \frac{\left(\pi_{1}\right)^{n}}{\left(\pi_{\alpha^{\prime}}\right)^{n-1}}$

and in the second chart by

$\hat{\omega}^{0}\left(u, v, \zeta, \widetilde{\zeta}, \pi_{A^{\prime}}\right)=u \pi_{0^{\prime}}+\zeta \pi_{1}$

$\hat{\omega}\left(u, v, \zeta, \widetilde{\zeta}, \pi_{A^{\prime}}\right)=\tilde{\zeta} \pi_{\rho}+v \pi_{1,}+\sum_{n=-1}^{\infty} b_{-n}(u, \zeta) \frac{\left(\pi_{0^{\prime}}\right)^{n+1}}{\left(\pi_{1},\right)^{n}}$

Computing the metric: Consider $(u, v, \zeta, \tilde{\zeta})$ as coordinates of a point in $G$. Let $(d u, d v, d \zeta, d \widetilde{\xi})$ be components of a tangent vector at $(u, v, \zeta, \widetilde{\zeta})$. According to the discussion in Sec. 1 , we get a section of the normal bundle to the section of $T$ labelled by $(u, v, \zeta, \widetilde{\zeta})$ by writing, in un-hatted coordinates,

$$
\begin{aligned}
V^{0}= & \pi_{0^{\prime}} d u+\pi_{1^{\prime}} d \zeta \\
V^{1}= & \pi_{\alpha^{\prime}} d \xi+\pi_{1^{\prime}} d v \\
& -\sum_{n=2}^{\infty}\left(\frac{\partial b_{n}}{\partial u} d u+\frac{\partial b_{n}}{\partial \zeta} d \zeta\right) \frac{\left(\pi_{1},\right)^{n}}{\left(\pi_{\alpha^{\prime}}\right)^{n-1}} .
\end{aligned}
$$

Assume $d \xi \neq 0$. Then, for a null vector, $\left(V^{\circ}\left(\pi_{A^{\prime}}\right)\right.$, $\left.V^{1}\left(\pi_{A^{\prime}}\right)\right)=(0,0)$ for some $\pi_{A^{\prime}}$. We must in fact have a zero at $\left(\pi_{0^{\prime}}, \pi_{1},\right)=(-d \zeta, d u)$. But then $V^{1}=0$ gives

$$
\begin{aligned}
0= & -d \zeta d \tilde{\zeta}+d u d v-\sum_{n=2}^{\infty} \frac{\partial b_{n}}{\partial u} \frac{(d u)^{n+1}}{(d \zeta)^{n-1}}(-1)^{n-1} \\
& -\sum_{n=2}^{\infty} \frac{\partial b_{n}}{\partial \zeta} \frac{(d u)^{n}}{(d \zeta)^{n-2}}(-1)^{n-1}, \\
0= & d u d v-d \zeta d \tilde{\zeta}+\frac{\partial b_{2}}{\partial \zeta} d u^{2} \\
& +\sum_{n=2}^{\infty}(-1)^{n}\left(\frac{\partial b_{n}}{\partial u}-\frac{\partial b_{n+1}}{\partial \zeta} \frac{(d u)^{n+1}}{(d \zeta)^{n-1}} .\right.
\end{aligned}
$$

But, recalling that $h(u+\xi \zeta, 1, \xi)=\sum_{n=-\infty}^{\infty} b_{n}(u, \zeta) \xi^{n}$, we conclude $\partial b_{n} / \partial u=\partial b_{n+1} / \partial \zeta$. Thus the power series vanishes and the conformal metric is given by

$$
d s^{2}=\kappa\left(d u d v-d \zeta d \xi+\frac{\partial b_{2}}{\partial \zeta} d u^{2}\right)
$$

where $\kappa$ is an arbitrary nonzero holomorphic function. We now show that the actual metric on $\zeta$ as defined by Penrose is obtained by taking $\kappa=2$. Let

$$
X:=\frac{\partial}{\partial u}-\frac{\partial b_{2}}{\partial \zeta} \frac{\partial}{\partial v}, \quad Y:=\frac{\partial}{\partial v} .
$$

$X$ and $Y$ define sections as in (10). For $X$ we have

$$
\begin{aligned}
& X^{0}=\pi_{0^{\prime}}, \\
& X^{\prime}=-\pi_{1}, \frac{\partial b_{2}}{\partial \zeta}-\sum_{n=2}^{\infty} \frac{\partial b_{n}}{\partial u}(u, \zeta) \frac{\left(\pi_{1},\right)^{n}}{\left(\pi_{0^{\prime}}\right)^{n-1}} .
\end{aligned}
$$

For $Y$ we have

$$
Y^{0}=0, \quad Y^{1}=\pi_{1} \cdot
$$

The section $X^{A}$ vanishes at $\pi_{A^{\prime}}=(0,1)$ while $Y^{A}$ vanishes at $\pi_{A^{\prime}}=(1,0)$. That $X^{A}(0,1)=0$ is not evident in (13), but one must remember "unhatted" coordinates are not valid for $\pi_{\alpha^{\prime}}=0$.

Let $x_{A^{\prime}}=(0,1), y_{A^{\prime}}=(1,0)$. Now $x^{A^{\prime}}=(1,0), y^{A^{\prime}}$ $=(0,-1)$. Then the Penrose inner product of $X$ and $Y$ is

$$
\begin{aligned}
& g(X, Y)=\frac{x_{A^{\prime}} y^{A^{\prime}} \mu\left(X^{A}, Y^{A}\right)}{\left(x_{A^{\prime}} \pi^{A^{\prime}}\right)\left(y_{A^{\prime}} \pi^{A^{\prime}}\right)}, \\
& x_{A^{\prime}} y^{A^{\prime}}=-1, \quad x_{A^{\prime}} \pi^{A^{\prime}}=-\pi_{0^{\prime}}, \quad y_{A^{\prime}} \pi^{A^{\prime}}=\pi_{1},
\end{aligned}
$$

and

$$
\mu\left(X^{A}(\pi), Y^{A}(\pi)\right)=X^{0}(\pi) Y^{1}(\pi)-X^{1}(\pi) Y^{0}(\pi)=\pi_{0} \pi_{1},
$$

So $g(X, Y)=(-1) \pi_{0}, \pi_{1}, /-\pi_{0}, \lambda_{1},=1$. On the other hand, if we simply substitute $X$ and $Y$ into (11), to obtain $g(X, Y)=1$ we find that we must take $k=2$, as asserted.

Now let $f(u, \zeta)$ be any entire function on $C^{2}$. We claim we can choose $h$ so that $\partial b_{2}(u, \zeta) / \partial \zeta=f(u, \zeta)$. If so, then we will have generated all metrics of the form

$$
d s^{2}=d u d v-d \zeta d \tilde{\zeta}+f(u, \zeta) d u^{2} .
$$

It is enough to show we can choose $h$ so as to make $b_{2}(u, \zeta)=g(u, \zeta)$, where $g$ is a given entire function. Write

$$
g(u, \zeta)=\sum_{n, m \geqslant 0} a_{m n} u^{m} \xi^{n},
$$

where the series converges everywhere. Define

$$
\phi(x, y):=\sum_{n_{v} m \geqslant 0} \frac{a_{m n}}{\left(\begin{array}{c}
m+n \\
n
\end{array}\right)} x^{m} y^{n}
$$

Then $\phi$ is an entire function of $x, y$. We then write,

$$
h\left(\omega^{0}, \pi_{0^{\prime}}, \pi_{1^{\circ}}\right):=\phi\left(\frac{\omega^{0}}{\pi_{\omega^{\circ}}}, \frac{\omega^{0}}{\pi_{1^{\prime}}}\right) \frac{\left(\pi_{1^{\prime}}\right)^{2}}{\pi_{0^{\circ}}} .
$$

Clearly $h$ is holomorphic on $C \times(D \cap \hat{D})$, and $h$ is homogeneous of degree 1 :

$$
\begin{aligned}
h(u+\zeta \xi, 1, \xi) & =\phi\left(\frac{u+\zeta \xi}{1}, \frac{u+\zeta \xi}{\xi}\right) \xi^{2} \\
& =\sum_{n, m \geqslant 0} \frac{a_{m n}}{\left(\begin{array}{c}
m+n \\
n
\end{array}\right)} \frac{(u+\zeta \xi)^{m+n}}{\xi^{n-2}}, \\
\frac{(u+\zeta \xi)^{m+n}}{\xi^{n-2}} & =\sum_{k=0}^{m+n}\left(\begin{array}{c}
m+n \\
k
\end{array}\right) u^{m+n-k} \zeta^{k} \xi^{k-n+2}
\end{aligned}
$$

so when everything is expanded in powers of $\xi$ for fixed $u$ and $\zeta$ the coefficient of $\xi^{2}$ is $\sum_{n, m \geqslant 0} a_{m n} u^{m} \zeta^{n}=g(u, \zeta)$ as desired. For the metric (15) one can directly show that the Ricci tensor vanishes; whether the Weyl tensor is self-dual or anti-self-dual depends on the choice of complex volume element $\epsilon_{a b c d^{*}}$. There is on $G$ a natural 
choice of $\epsilon$ due to the existence of a natural spinor structure on $\mathcal{G}$. In the coordinates $\left(z^{\mu}\right)=(u, v, \zeta, \tilde{\zeta})$ used above, $\epsilon$ is specified by $\epsilon_{0123}=-i$. With this choice the space is right-flat, i.e., ${ }^{*} C_{a b c d}=-i C_{a b c d}$.

\section{ACKNOWLEDGMENT}

One of us (D. L.) is grateful to K. P. Tod for an ex- planation of some of the details of Penrose's construction.

${ }^{1}$ R. Penrose, Gen. Rel. Grav. 7, 31 (1976).

${ }^{2}$ K. Kodaira, Am. J. Math. 85, 79 (1963).

${ }^{3}$ K. Kodaira, Ann. Math. 75, 146 (1962).

${ }^{4}$ E. T. Newman, J. R. Porter, and K. P. Tod, Preprint.

${ }^{5}$ J.F. Plebański, J. Math. Phys. 16, 2396 (1975). 